\title{
Bromatologia e contaminação com fumonisina e aflatoxina em rações utilizadas na piscicultura da região de Londrina, Estado do Paraná, Brasil
}

\section{Bromatology and fumonisin and aflatoxin contamination in aquaculture feed of the region of Londrina, State of Paraná, Brazil}

\author{
Elisabete Hiromi Hashimoto ${ }^{1}$; Maria Angela do Santos ${ }^{1}$; \\ Elisabete Yurie Sataque $\mathrm{Ono}^{3}$; Carmino Hayashi ${ }^{4}$; \\ Ana Paula Frederico Rodrigues Loureiro Bracarense ${ }^{5}$; Elisa Yoko Hirooka ${ }^{6 *}$
}

\begin{abstract}
Resumo
Com o intuito de suprir a demanda e o progresso na piscicultura, o setor industrial investiu na produção de rações, cuja formulação depende de ingredientes constituídos de grãos susceptíveis à contaminação por fungos micotoxigênicos. O trabalho visou avaliar a composição química (qualidade nutricional), aliada à susceptibilidade ao risco de contaminação por micotoxinas (aflatoxina e fumonisina), em 42 amostras de ração, pertencentes a cinco marcas comerciais, utilizadas nos pesqueiros da Região de Londrina-PR. A análise da composição química indicou qualidade nutricional adequada das rações, não se constatando diferença significativa na composição bromatológica geral entre os tipos de rações extrusada e peletizada ( $\mathrm{p}>0,05)$. Os níveis de aflatoxina variaram desde não detectável a 15,60 ng/g, com $61,90 \%$ de amostras em níveis <4ng/g, indicando estar dentro da recomendação nacional, fixada em 20 ng/g. Em relação a fumonisina (não detectado a $11,22 \mu \mathrm{g} / \mathrm{g}$ ), 76,20\% das amostras apresentaram níveis $<4 \mu \mathrm{g} / \mathrm{g}$. Não se constatou diferença entre ração peletizada e extrusada para os níveis destas micotoxinas ( $p>0,05)$, porém a co-ocorrência de aflatoxina com fumonisina em 23,8\% das rações, sugeriu risco de sinergismo tóxico, apontando a importância de monitoramento no controle de micotoxinas na dieta de peixes. Por outro lado, considerando a constante renovação de rações nos pesqueiros, pode-se inferir uma baixa possibilidade do incremento de aflatoxina e fumonisina devido a armazenagem local, devendo o ponto crítico ser direcionado ao estágio de produção de matéria prima no campo e pré-processamento, independentemente de marca ou diferenças nutricionais.
\end{abstract}

Palavras-chave: Aflatoxinas, fumonisinas, ração, piscicultura.

Abstract
With intention to supply the demand and progress of aquaculture, the industrial sector invested in the
production of feed, whose formulation depends on grains susceptible for mycotoxigenic fungi. The
objective of this work was to evaluate the chemical composition (nutricional quality) associated with the
rmacêutica, Mestre e Doutoranda em Ciência de Alimentos - Depto. Tecnologia de Alimentos e Medicamentos- Centro de
cente do Depto. de Bioquímica - Centro de Ciências Exatas- Universidade Estadual de Londrina.
cente do Depto. de Aqüicultura - Centro de Ciências Biológicas- Universidade Estadual de Maringá.
cente do Depto. de Medicina Veterinária Preventiva - Hospital Veterinário - Universidade Estadual de Londrina.
tonte do Depto. Tecnologia de Alimentos e Medicamentos- Centro de Ciências Agrárias - Universidade Estadual de Londrina.


Hashimoto, E. H. et al.

risk of mycotoxin contamination (aflatoxin and fumonisin) in 42 feed samples, belonging to five commercial industries, and used in fishing activity of the Region of Londrina-PR. The analysis of chemical composition indicated adequate nutritional quality of feeds, without significant difference concerning bromatological composition between extruded and pellet feeds ( $p>0.05)$. The aflatoxin levels ranged from non-detectable to $15.60 \mathrm{ng} / \mathrm{g}$, where $61.90 \%$ showed < $4 \mathrm{ng} / \mathrm{g}$ levels, which are in accordance with the Brazilian guideline $(20 \mathrm{ng} / \mathrm{g}$ ). Related to fumonisin (n.d. to $11,221 / 4 \mathrm{~g} / \mathrm{g}$ ), $76.20 \%$ samples were into the levels $<41 / 4 \mathrm{~g} / \mathrm{g}$. There was no significant difference between pellet and extruded feeds concerning mycotoxin contamination ( $p>0,05$ ), but the aflatoxin/fumonisin co-occurrence in $23.8 \%$ feed samples suggested risk of toxic synergism, emphasizing the importance of mycotoxin monitoring in fish feeding quality. Taking into account the continuous renewal of feed in the fishing ones, there is low possibility of aflatoxin/fumonisin production due the storage, therefore the critical point should be targeted on crude material at field to pre-processing stage, independently of mark or nutritional differences.

Key words: Aflatoxins, fumonisins, feed, aquaculture.

\section{Introdução}

A piscicultura brasileira experimentou crescimento substancial na última década, atraindo as indústrias de ração com diversificação na qualidade e conseqüente alta nos preços (EMATER, 2002; ARAGÃO, 2002). O fato tornou 50 a $70 \%$ da produção de peixe dependente de fatores como a qualidade e o custo, direcionando os nutricionistas ao estudo sobre viabilidade econômica da criação (EL-SAYED, 1999; FURUYA, 2001).

As rações comerciais devem atender o requerimento nutricional principalmente de espécies tropicais de água doce, exemplificando-se o pacu (Piractus mesopotamicus), o piau (Leporinus sp.) e as tilápias (Oreochromis sp.) entre as espécies mais cultivadas (EMATER, 2002). A inadequação nutricional causa baixa taxa de crescimento e conversão, além de tolerância à manipulação e transporte, aliados à alta deformidade anatômica e sinais clínicos de deficiências vitamínica e mineral e com maior incidência de doença e morte (KUBITZA \& CYRINO, 1999; LALL, 1988). Além do teor nutricional, a qualidade da ração depende de estabilidade na água e da aceitabilidade pelo peixe e dos atributos físicos como tamanho, forma, dureza, textura e capacidade de flutuação. No contexto, a popularidade de rações extrusadas, embora de maior custo, se deve a facilidade no manejo e produtividade, em relação à modalidade peletizada (MEYER, 1999).

A qualidade das rações depende ainda de controle sanitário de matéria-prima básica de origem vegetal incorporada na formulação, devido a susceptibilidade aos fungos micotoxigênicos. Milho, soja, arroz e trigo são contaminados freqüentemente por Aspergillus spp. e Fusarium spp., produtores de aflatoxinas e fumonisinas, respectivamente (ABOUZIED \& PESTKA, 1994; HIROOKA et al., 1996; ONO et al., 1999, ONO et al., 2002, ONO \& HIROOKA, 2002). A aflatoxina $B_{1}\left(A_{F B}\right)$, reconhecidamente carcinogênica, teratogênica e mutagênica, se enquadra entre carcinógeno primário de Classe 1 (IARC, 1993). $\mathrm{FB}_{1}$, a mais importante no grupo de fumonisinas, causa leucoencefalomalácia eqüina, edema pulmonar suína e imunossupressão aviária, além de provável correlação com câncer esofágico humano (HARRISON et al., 1990; NORRED \& VOSS, 1994; MARASAS, 1995; CREPPY, 2002). $O$ fato requer legislação a nível internacional envolvendo a participação conjunta de 'Food and Agriculture Organization' e 'World Health Organization' através de 'Joint FAO/WHO Expert Committee of Food Additives' - JECFA (WHO, 1998; FAO, 2001).

Desconhece-se o risco de contaminação humana indireta através do consumo de peixes (SMITH \& MOSS, 1985). Não obstante, a bioacumulação ter sido fenômeno comum nesta espécie, não se podendo descartar a repercussão da contaminação com micotoxinas na dieta à saúde humana (PLAKAS et al.,1991). A produção brasileira elevou-se de 6,6 mil toneladas em 1995 para 17,5 mil toneladas de peixes fluviais em 2001, sendo $62 \%$ destinadas aos pesque- 
pagues, $26 \%$ às indústrias, $10 \%$ consumidos diretamente e $2 \%$ comercializadas em feiras (EMATER, 2002). A comercialização através de pesque-pague quadruplicou no Estado do Paraná, com incremento anual de 1,1 mil em 1995, para 4,3 mil toneladas em 2001 (ARAGÃO, 2002). Embora o mercado nacional apresenta dependência de capturas e de importação de pescados, o Brasil obteve pela primeira vez um superávit de US\$22,6 milhões na balança comercial em 2001, principalmente com a venda de atuns (HOLANDA et al., 2000; BRASIL, 2001). Estes fatos indicam tendência ávida de expansão da área visando a comercialização, merecendo atenção especial no contexto de segurança alimentar na cadeia produtiva de peixes.

A atividade de pesque-pague envolvendo a criação de tilápias se destaca na periferia da cidade de Londrina no interior paranaense, resultante da crescente expansão urbana (ARAGÃO, 2002). O benefício do lazer, em contrapartida, pode trazer risco de contaminação indireta com micotoxinas, oriundas de bioacumulação em peixes, devendo-se complementar a avaliação da qualidade nutricional de rações, com o fator segurança e sanidade .

Diante de perspectivas promissoras da piscicultura, aliada à escassez de informações sobre micotoxinas em rações de peixe, avaliou-se a composição bromatológica relacionada com riscos de contaminação com aflatoxina e fumonisina em rações utilizadas na criação de Tilápia do Nilo (Oreochromis niloticus) nos pesqueiros de Londrina, Estado do Paraná-BR.

\section{Material e métodos}

\section{Amostras de ração}

42 amostras de ração, sendo 31 extrusadas e 11 peletizadas, foram adquiridas de cinco pesqueiros no Município de Londrina, Região Norte do Paraná, no período de março a julho/2001. Todas as amostras pertenceram a cinco marcas comerciais (A, B, C, D e E). As 31 amostras extrusadas foram distribuídas em 8 amostras da marca A, 11 marca B, 3 marca C, 2 marca D e 7 marca E. As 11 amostras peletizadas consistiram de 8 de marca A, 2 marca B e 1 marca E. Na amostragem considerou-se a renovação do estoque ( 1 a 3 sacos de $25-30 \mathrm{Kg} / \mathrm{semana}$ ), procedendo coleta mensal em março (10), abril (10), maio (11), junho (11), num total de $2 \mathrm{~kg}$ de ração por amostra (retirada em três pontos do saco: fundo, meio e superfície). Após homogeneização, as amostras foram embaladas, vedadas em sacos plásticos, enviadas ao Laboratório de Tecnologia de Alimentos e Medicamentos da Universidade Estadual de Londrina e mantidos a $4^{\circ} \mathrm{C}$ até a análise, realizada dentro de um mês, onde procedeu-se o quarteamento para redução final de 400-500g.

\section{Composição bromatológica}

A avaliação de composição química consistiu na determinação de porcentagem de proteínas, lipídios e resíduos minerais em base seca, realizadas conforme descrito em Normas do Instituto Adolfo Lutz (1985). O teor de umidade foi determinado pelo método de infravermelho (OHAUS®).

\section{Determinação de fumonisinas}

As fumonisinas foram quantificadas pela cromatografia líquida de alta eficiência - CLAE, segundo Shephard et al. (1990), modificado por Ueno et al. (1993). As fumonisinas foram extraídas com metanol:água $(3: 1, v / v)$, derivatizadas com oftaldialdeído-OPA e injetado em sistema CLAE isocrático de fase reversa da Shimadzu, equipado com bomba (LC-10 AD), detector de fluorescência (RF 535) e coluna $\mathrm{C}_{18}$ - CLC-ODS (M) (4,6x 250mm). Os comprimentos de onda de excitação e emissão foram de $335 \mathrm{~nm}$ e $450 \mathrm{~nm}$, respectivamente. A fase móvel consistiu de $\mathrm{CH}_{3} \mathrm{OH}-\mathrm{NaH}_{2} \mathrm{PO}_{4} 0,1 \mathrm{~mol} / \mathrm{L}$ (80:20, v/v) pH 3,3, ajustado para o fluxo de $1,0 \mathrm{~mL} /$ min. Os limites de quantificação para $\mathrm{FB}_{1}$ e $\mathrm{FB}_{2}$ foram de $50 \mathrm{ng} / \mathrm{g}$ e $80 \mathrm{ng} / \mathrm{g}$, respectivamente. 
Determinação de aflatoxinas $B_{1}, B_{2}, G_{1}$ e $G_{2}$

A determinação simultânea de aflatoxinas $\left(\mathrm{B}_{1}\right.$, $B_{2}, G_{1}$ e $G_{2}$ ) foi realizada pela cromatografia em camada delgada - CCD (SOARES \& RODRIGUES-AMAYA, 1996). A fase móvel consistiu de tolueno: acetato de etila: ácido fórmico (60:40:0,5 v/v) e reveladas sob luz ultravioleta com comprimento de onda longa ( $366 \mathrm{~nm})$. Os limites de detecção para $\mathrm{AFB}_{1}$ foi de 2,0ng/g e o limite de quantificação de $4,0 \mathrm{ng} / \mathrm{g}$.

\section{Análise estatística}

O dimensionamento de coleta para 42 amostras baseou-se na pré-amostragem de 10 amostras de rações, onde se obteve-se média e variância de $5,98 \mathrm{ng} / \mathrm{g} \pm 2,796$ para aflatoxinas e $3,05 \mu \mathrm{g} / \mathrm{g} \pm 1,231$ para fumonisinas. A amostragem mínima calculada foi de 38 a nível de significância de 5\%, porém fixou-se a coleta em 42 amostras, para garantir a análise. As diferenças na composição bromatológica e contaminação por micotoxinas em rações extrusadas e peletizadas, pertencentes a cinco marcas, foi realizada através do Teste de Tukey, para comparação múltipla entre as médias com significância de 5\%, utilizando o programa "Software Statistica", versão 5.0.

\section{Resultados e Discussão}

A Tabela 1 mostra que o teor de umidade das rações pertencentes a cinco marcas comerciais, coletadas nos pesqueiros variou de 3,6 a 14,5\%, resultando na variação média de 8,83 a $11,50 \%$ para as amostras extrusadas e 9,7 a $12,3 \%$ para as peletizadas. Considerando que a umidade máxima permitida por 'Aquaculture Developing and Control Program'- ADCP/FAO (1987; 2000) é de 10 a 13\%, a extensa faixa de variação observada na ração (Tabela 1) provavelmente decorreu de alta umidade relativa no local de armazenagem, associado ao curto tempo de permanência, não excedente a uma semana. $\mathrm{O}$ binômio umidade/temperatura constitui fator crítico no crescimento fúngico e produção de micotoxinas (ONO, et al., 1999), indicando a necessidade de se manter um manter pequeno estoque de ração, permitindo giro rápido.

Baseado nos dados de umidade, calculou-se os teores de cinzas, proteínas e extrato etéreo em base seca, conforme apresentados na Tabela 1. A análise de variância não constatou diferença significativa na composição bromatológica das médias em geral entre os tipos de ração extrusada e peletizada $(\mathrm{p}>0,05)$. As rações extrusadas e peletizadas apresentaram teores médios de 7,60; 31,98; 3,95\% e 7,35; 29,87; $3,49 \%$ para cinzas, proteínas e extrato etéreo, respectivamente. Outrossim, centrando-se a análise no tipo extrusado, a marca C $(35,08 \%)$ e D $(34,39 \%)$ apresentaram maior porcentagem média de proteína bruta $(\mathrm{p}<0,05)$.

$\mathrm{O}$ teor de nutrientes requeridos pelos peixes depende da idade, sendo que os valores recomendados pela ADCP/FAO (1987) para tilápia variam de 6 a 10\% de lipídeos, 30 a 50\% de proteínas (correspondente a $4,1 \%$ de lisina e $1,7 \%$ de metionina + cistina), $25 \%$ de carboidratos e 8 a $10 \%$ de fibras. Assim, os peixes requerem rações com maior porcentagem de proteína do que outras espécies, estando a exigência de proteína bruta para Tilápia do Nilo, na faixa de 28 a $32 \%$ (FURUYA, 2001), ou $30 \%$ conforme recomendações do ADCP/FAO (1987). Neste contexto, as rações analisadas com o teor protéico entre 28,1 a $35,24 \%$ atenderam os requisitos propostos (Tabela 1).

O teor lipídico variou de 3,02\% (marca A, peletizada) a $4,63 \%$ (C, extrusada), satisfazendo as especificações constantes nas respectivas embalagens comerciais (mínimo 3-4\% e máximo 9\%). Embora estes valores estejam abaixo do limite de 6-10\%, recomendado pela ADCP/FAO (1987), o extrato etéreo pode variar conforme a relação proteína:energia e tipo de dieta, já que a deficiência impede a retenção adequada de proteínas, enquanto o excesso eleva o conteúdo lipídico visceral e corporal. Atenção especial deve 
ser dada a ácidos graxos essenciais, sendo que a tilápia requer aproximadamente $0,5 \%$ de ácido linoleico (FURUYA, 2001).

As rações extrusadas e peletizadas com teor médio de resíduos minerais de $7,60 \%$ e $7,35 \%$, respectivamente (Tabela 1), apresentaram-se dentro do limite estabelecido pelas empresas, que recomendam o máximo de 9,7-14,0\%, com ênfase ao cálcio (máximo de 3,0-4,5\%).Todavia, com relação à fósforo (recomendação mínima de 0,5-1,2\%), geralmente ocorre suplementação na dieta, já que a deficiência em tilápias causa redução no crescimento e conversão alimentar, retenção de macrominerais e aumento na gordura visceral (LALL, 1988).

Tabela 1. Composição bromatológica em base seca (teor de umidade, cinzas, proteínas e extrato etéreo) de rações extrusadas da marca A, B, C, D e peletizada demarca A, B e E.

\begin{tabular}{|c|c|c|c|c|c|c|}
\hline \multirow[b]{2}{*}{ Ração } & \multirow[b]{2}{*}{ Marcas } & \multirow[b]{2}{*}{$\mathbf{n}$} & \multicolumn{4}{|c|}{ Porcentagem em g\%, bs* (média) } \\
\hline & & & Umidade & Cinza* & Proteína* & Extrato etéreo* \\
\hline \multirow{5}{*}{ Extrusada } & $\mathbf{A}$ & 8 & $\mathbf{3 , 8 0 - 1 1 , 2}(8,83 \pm 3,03)$ & $\mathbf{6 , 9 5 - 8 , 0 8}(7,57 \pm 0,64)^{\mathrm{a}}$ & $\mathbf{2 8 , 1 0 - 3 0 , 2 4}(29,44 \pm 0,95)^{\mathrm{c}}$ & $\mathbf{3 , 7 0 - 4 , 0 9}(3,89 \pm 0,160)^{\mathrm{a}}$ \\
\hline & B & 11 & $\mathbf{3 , 6 0 - 1 4 , 5 0}(9,13 \pm 3,49)$ & $\mathbf{6 , 6 2 - 7 , 7 5}(7,18 \pm 0,42)^{\mathrm{b}}$ & $\mathbf{2 9 , 9 8 - 3 2 , 6 8}(31,14 \pm 1,49)^{\mathrm{b}}$ & $\mathbf{3 , 2 7 - 3 , 8 0}(3,54 \pm 0,25)^{\mathrm{b}}$ \\
\hline & C & 3 & $\mathbf{1 0 , 4 0 - 1 3 , 4 0}(11,50 \pm 1,65)$ & $\mathbf{8 , 1 0 - 8 , 4 7}(8,28 \pm 0,17)^{\mathrm{a}}$ & $\mathbf{3 4 , 8 3 - 3 5 , 2 4}(35,08 \pm 0,22)^{\mathrm{a}}$ & $\mathbf{4 , 5 2 - 4 , 6 3}(4,57 \pm 0,05)^{\mathrm{a}}$ \\
\hline & D & 2 & $\mathbf{1 1 , 0 0 - 1 1 , 5 0}(11,25 \pm 0,35)$ & $\mathbf{7 , 4 6 - 7 , 6 0}(7,53 \pm 0,10)^{\mathrm{b}}$ & $\mathbf{3 4 , 1 - 3 4 , 6 7}(34,39 \pm 0,40)^{\mathrm{a}}$ & $\mathbf{3 , 3 1 - 3 , 4 6}(3,39 \pm 0,11)^{\mathrm{b}}$ \\
\hline & $\mathbf{E}$ & 7 & $\mathbf{7 , 5 0 - 1 3 , 3 0}(9,61 \pm 2,24)$ & $7,05-7,99(7,46 \pm 0,45)^{\mathrm{b}}$ & 29,14-30,18 $(29,84 \pm 0,41)^{\mathrm{b}, \mathrm{c}}$ & $\mathbf{4 , 1 6}-\mathbf{4 , 5 5}(4,38 \pm 0,20)^{\mathrm{a}}$ \\
\hline \multirow[t]{2}{*}{ MÉDIA } & & & $10,06^{\mathrm{a}}$ & $7,60^{a}$ & $31,98^{a}$ & $3,95^{a}$ \\
\hline & $\mathbf{A}$ & 8 & $\mathbf{1 0 , 0 3 - 1 2 , 9}(11,31 \pm 1,24)$ & $\mathbf{6 , 9 0 - 8 , 1 9}(7,57 \pm 0,64)^{\mathrm{a}}$ & $\mathbf{2 8 , 1 - 3 0 , 2 4}(29,44 \pm 0,95)^{\text {b }}$ & $\mathbf{3 , 0 2 - 3 , 3 6}(3,19 \pm 0,158)^{\mathrm{b}}$ \\
\hline \multirow[t]{2}{*}{ Peletizada } & B & 2 & $\mathbf{7 , 8 6 - 1 1 , 5 4}(9,7 \pm 2,60)$ & $\mathbf{7 , 0 1 - 7 , 9 8}(7,49 \pm 0,64)^{\mathrm{a}}$ & $\mathbf{3 0 , 5}(30,05 \pm 0,00)^{\mathrm{a}}$ & $\mathbf{3 , 1 6 - 3 , 4 1}(3,29 \pm 0,176)^{\mathrm{b}}$ \\
\hline & $\mathbf{E}$ & 1 & $\mathbf{1 2 , 3}(12,3)$ & $\mathbf{6 , 9 9}(6,99)^{\mathrm{a}}$ & $\mathbf{3 0 , 1 4}(30,14)^{\mathrm{a}}$ & $4,00(4,00)^{\mathrm{a}}$ \\
\hline MÉDIA & & & $11,10^{a}$ & $7,35^{a}$ & $29,87^{a}$ & $3,49^{a}$ \\
\hline
\end{tabular}

Nível de significância: $\mathrm{p}<0,05$

* : base seca

$\mathrm{n}=\mathrm{n}^{\mathrm{o}}$ de amostras

A Tabela 2 mostra os resultados da análise de micotoxinas, com aflatoxina constatada nas marcas de ração $\mathrm{A}, \mathrm{B}, \mathrm{C}$ e $\mathrm{E}$ (n.d. a 15,60 ng/g), sendo $\mathrm{AFB}_{1}$ detectada em maior frequiência e concentração (n.d. a $13,13 \mathrm{ng} / \mathrm{g}$ ). Fumonisina foi verificada em quatro das cinco marcas de rações (n.d. a 11,22 $\mu \mathrm{g} / \mathrm{g}$ ), com exceção do tipo extrusado marca $\mathrm{D}$, confirmando a unanimidade do emprego de milho como ingrediente de ração. Neste último grupo também não se detectou aflatoxina (Tabela 2), em adição a melhor qualidade nutricional, representada pelo teor protéico de 34,39\% (Tabela 1). A marca C apresentou elevado conteúdo protéico com teor médio de $35,08 \%$ (Tabela 1), porém no grupo ocorreu maior teor médio de $8,21 \mathrm{ng} / \mathrm{g}$ de aflatoxinas e fumonisinas de $6,404 \mu \mathrm{g} /$ g (Tabela 2). Não obstante, deve-se considerar a pequena amostragem realizada, sendo que a marca C, constituída de 3 amostras, continham duas amostras com menos de 4ng/g e uma com 15,60ng/g de aflatoxina, elevando o teor médio.
A marca A (total de 16 amostras) apresentou maior freqüência de amostras positivas para aflatoxina, compreendendo 5 amostras extrusadas com média de 3,28ng/g (n.d. a 9,16ng/g,) e 2 peletizadas com teor médio de $1,28 \mathrm{ng} / \mathrm{g}$ (n.d. a $4,59 \mathrm{ng} / \mathrm{g}$,). Em relação a fumonisina, além de marca A com 5 amostras extrusadas e 3 peletizadas positivas, destacou-se a marca $\mathrm{E}$ com positividade total de 7 extrusadas e 1 peletizadas (Tabela 2).

Os níveis de aflatoxinas nas rações analisadas (Tabela 2), apresentaram-se dentro dos limites permitidos pela legislação brasileira, conforme a Portaria $n^{\circ} .183$ de 21/3/1996 do Ministério da Agricultura, Pecuária e Abastecimento, que estabelece níveis de aflatoxina $\left(B_{1}+B_{2}+G_{1}+G_{2}\right)$ em 20ng/g, baseado na Resolução n ${ }^{\circ}$. 54-1994 do Mercosul (MERCOSUL, GMC/ Res. 56/1994). Com relação a fumonisinas, ainda não existem limites legais estabelecidos, mas o 'Mycotoxin Committee of the American Association of Veterinary Laboratory 
Diagnosticians' recomendou níveis máximos de 5, 10,50 e $50 \mu \mathrm{g} / \mathrm{g}$ para rações destinadas a eqüinos, suínos, bovinos e aves, respectivamente (MUNKVOLD \& DESJARDINS, 1997). Em seres humanos, a FAO (2001) propôs ingestão diária máxima tolerada de fumonisinas de $2 \mu \mathrm{g} / \mathrm{Kg}$ de peso corpóreo. Por outro lado, limitou as aflatoxinas em 19ng por indivíduo, nos países onde vigora o limite máximo permitido de 20ng/g (WHO, 1998).

Tabela 2. Aflatoxina(ng/g de ração) e fumonisina $(\mu \mathrm{g} / \mathrm{g})$ em rações de peixe extrusadas da marca A, B, C, D e peletizada demarca A, B e E.

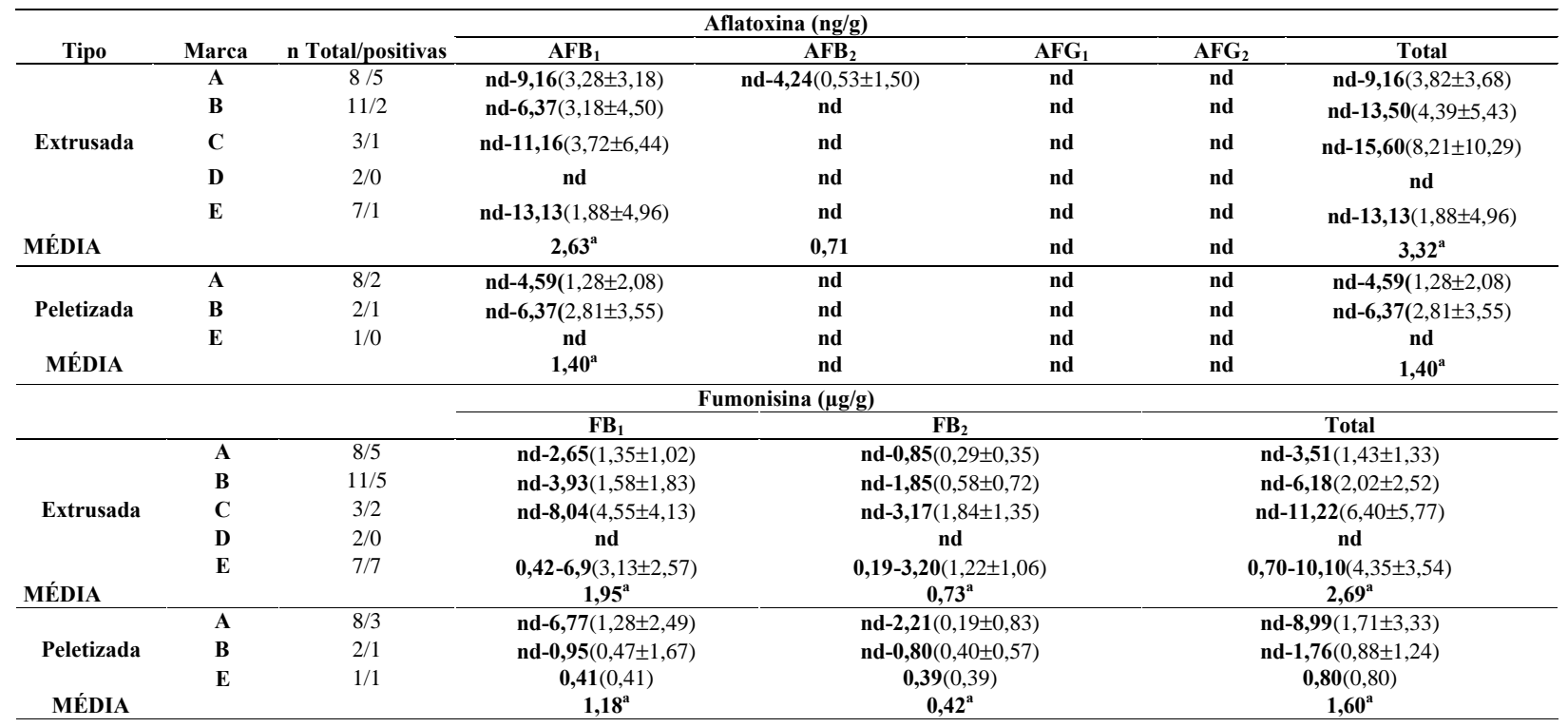

Nível de significância: $\mathrm{p}<0,05$

Limite de quantificação: $\mathrm{AFB}_{1}, 4 \mathrm{ng} / \mathrm{g} ; \mathrm{FB}_{1}, 50 \mathrm{ng} / \mathrm{g} ; \mathrm{FB}_{2}, 80 \mathrm{ng} / \mathrm{g}$ nd: não detectado

$\mathrm{n}=\mathrm{n}^{\mathrm{o}}$ de amostras

A Tabela 3 apresenta os níveis de micotoxinas analisadas em 42 amostras. Salienta-se a constatação de aflatoxina em $61,9 \%$ de amostras, em níveis abaixo de 4 ng/g (Tabela 3). As amostras com níveis de aflatoxina acima deste valor $(38,10 \%)$, com o máximo situado em 15,60 ng/g, indicaram que as rações analisadas estiveram dentro da recomendação nacional, fixada em $20 \mathrm{ng} / \mathrm{g}$ (MICOTOXINAS, 2001). Considerando-se o limite de 10ng/g de ração, imposto pela maioria dos membros da comunidade importadora da União Européia (FAO, 2001), podese inferir que $88,1 \%$ dos valores estiveram acima de $10 \mathrm{ng} / \mathrm{g}$ (Tabela 3). Em relação a fumonisinas, $76,20 \%$ das amostras apresentaram níveis abaixo de $4 \mu \mathrm{g} / \mathrm{g}$ (Tabela 3). Estes dados sugerem um perfil promissor para o controle posterior através de um monitoramento planejado, tendo em vista que se recomenda no máximo $5 \mu \mathrm{g} / \mathrm{g}$ para eqüino, considerado um animal muito sensível (MUNKVOLD \& DESJARDINS, 1997).

Apesar do baixo nível observado na maioria das amostras (Tabela 3), a Figura 1 mostrou que a coocorrência de $\mathrm{AFB}_{1}$ com fumonisina ocorreu nas marcas A em 31,25\%; B, 30,77\% e E, $12,5 \%$ das amostras. $\mathrm{O}$ fato merece atenção especial, já que a ingestão simultânea pode resultar na potencialização do efeito tóxico. A co-ocorrência de $\mathrm{AFB}_{1}$, considerado um potente iniciador de câncer hepático, com fumonisina $\mathrm{B}_{1}$ promotora de câncer, vem sendo cautelosamente discutida. 
Tabela 3. Níveis de aflatoxinas $B_{1}+B_{2}+G_{1}+G_{2}\left(n g / g\right.$ de ração) e fumonisinas $B_{1}+B_{2}(\mu g / g$ de ração) em amostras de rações de peixe.

\begin{tabular}{cccc}
\hline \multicolumn{2}{c}{ Aflatoxinas } & \multicolumn{2}{c}{ Fumonisinas } \\
\hline $\mathbf{B}_{\mathbf{1}}+\mathbf{B}_{2}+\mathbf{G}_{\mathbf{1}}+\mathbf{G}_{\mathbf{2}}(\mathbf{n g} / \mathbf{g})$ & no. de amostras $(\%)$ & $\mathbf{F B}_{\mathbf{1}}+\mathbf{F B}_{\mathbf{2}}(\boldsymbol{\mu} \mathbf{g} / \mathbf{g})$ & $\mathbf{n o .}$ de amostras $(\%)$ \\
\hline $\mathbf{n d - 4}$ & $\mathbf{2 6}(61,90)$ & $\mathbf{n d - 2}$ & $\mathbf{2 6}(61,90)$ \\
$\mathbf{4 - 6}$ & $\mathbf{7}(16,70)$ & $\mathbf{2 - 4}$ & $\mathbf{6}(14,30)$ \\
$\mathbf{6 - 8}$ & $\mathbf{1}(2,40)$ & $\mathbf{4 - 6}$ & $\mathbf{4}(9,5)$ \\
$\mathbf{8 - 1 0}$ & $\mathbf{3}(7,10)$ & $\mathbf{6 - 8}$ & $\mathbf{3}(7,1)$ \\
$\mathbf{1 0 - 1 2}$ & $\mathbf{1}(2,40)$ & $\mathbf{8 - 1 0}$ & $\mathbf{1}(2,4)$ \\
$\mathbf{1 2 - 1 4}$ & $\mathbf{3}(7,10)$ & $\mathbf{1 0 - 1 2}$ & $\mathbf{2}(4,8)$ \\
$\mathbf{1 4 - 1 6}$ & $\mathbf{1}(2,40)$ & & \\
\hline
\end{tabular}

Limite de quantificação: $\mathrm{AFB}_{1}, 4 \mathrm{ng} / \mathrm{g} ; \mathrm{FB}_{1}, 50 \mathrm{ng} / \mathrm{g} ; \mathrm{FB}_{2}, 80 \mathrm{ng} / \mathrm{g}$ nd: não detectado.

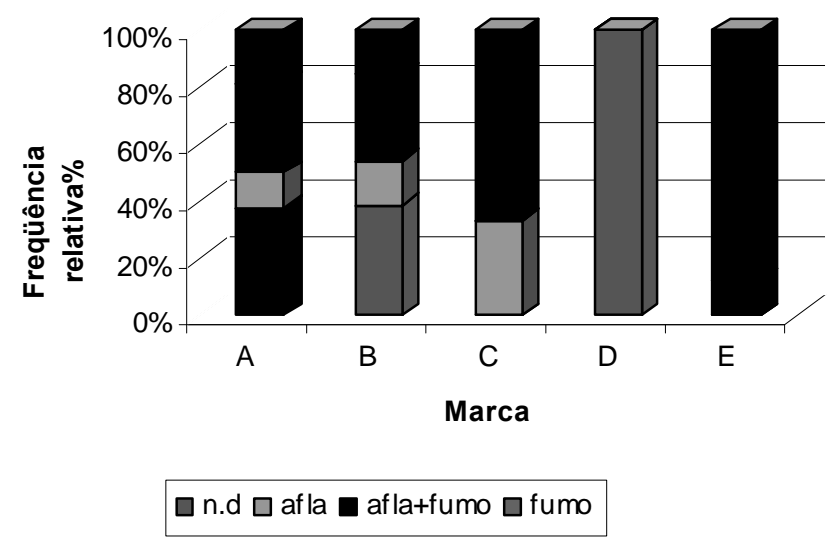

Figura 1. Co-ocorrência e freqüência relativa de aflatoxinas e fumonisinas marcas de ração A, B, C, D e E.

A Figura 2 compara os níveis de contaminação por aflatoxina (Figura 2A) e fumonisina (Figura 2B), considerando diferença no processo de produção. Para ração peletizada aplicou-se vapor à $90-100^{\circ} \mathrm{C}$, seguida de compactação e secagem, enquanto que na extrusada procedeu-se o processamento à 115$140{ }^{\circ} \mathrm{C}$, seguida de extrusão.

O Teste de Tukey indicou que não houve diferença significativa entre os dois tipos de rações, quanto a contaminação por aflatoxina ou fumonisina $(p>0,05)$, contrariando a expectativa (Figura 2). Busser \& Abbas (2002) reduziram 33\% do teor de $\mathrm{AFB}_{1}$ em sementes de algodão, fixando o tempo de extrusão e variando a temperatura de 104 e $160{ }^{\circ} \mathrm{C}$. Cazzaniga et al. (2001), comparando o efeito de extrusão, conseguiram eliminar $95 \%$ de deoxinivalenol (DON) e $10-25 \%$ de $\mathrm{AFB}_{1}$ em farinha de milho, enquanto que Jackson et al (1996) reduziram fumonisinas 25 a $30 \%$ com temperatura de $125^{\circ} \mathrm{C}$ e $90 \%$ à $175^{\circ} \mathrm{C}$, no processamento de alimentos à base de milho. Neste contexto, a fumonisina ocorreu em $61,29 \%$ das amostras extrusadas e 45,45\% das amostras peletizadas (Tabela 2), em comparação à contaminação quase que total, detectada no milho 'in natura' por Hirooka et al. (1996), Ono et al. (1999) e Ono et al. (2002).

Assim, embora o processo de extrusão seja mais drástico do que a peletização, os dados da Figura 2 mostraram que a qualidade da matéria prima utilizada no preparo da ração foi um fator decisivo na contaminação por micotoxinas. Todavia, para a confirmação do real ponto crítico, deve-se proceder 
o rastreamento desde o campo, em cada matéria prima. Em relação ao milho, que constitui o principal ingrediente na ração, trabalhos prévios demonstraram contaminação freqüente por Fusarium spp. (HIROOKA et al., 1996; ONO et al., 1999, 2002). Consequentemente, os resultados (Tabela 3, Figura 1 e 2) indicaram a provável contaminação por fumonisina oriunda do milho, enquanto que a aflatoxina foi devido ao uso de lote contendo milho velho, ou contaminação na recepção ou falha na seleção de matéria-prima na indústria.

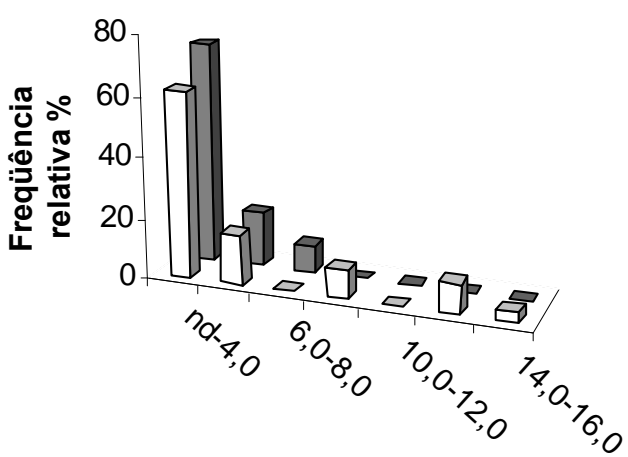

Aflatoxinas (ng/g)

\section{$\square$ Extrusada $\square$ Peletizada}

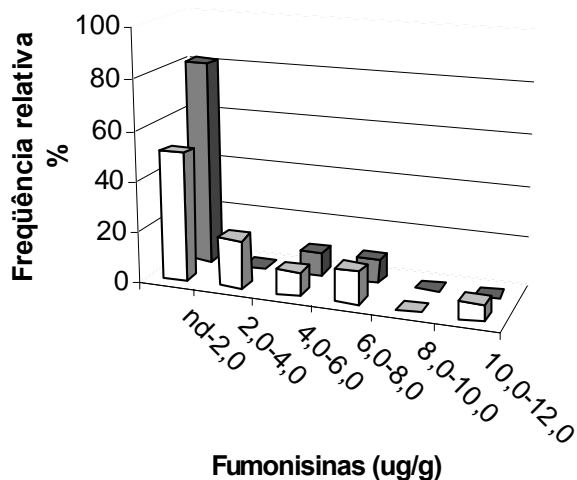

$\square$ Extrusada $\square$ Peletizada

Figura 2. Frequiência relativa de Aflatoxinas (A) e Fumonisinas (B) em amostras de rações extrusadas e peletizadas.
Considerando a constante renovação de estoques de rações nos pesqueiros analisados (1 a 3 sacos/ semana), pode-se inferir uma baixa possibilidade do incremento de aflatoxina e fumonisina devido a armazenagem local, estando o ponto crítico situado anível de campo e pré-processamento, independentemente de marca ou diferenças nutricionais.

\section{Conclusão}

As rações extrusadas e peletizadas apresentaram teor médio de umidade, cinzas, proteínas e extrato etéreo similares, indicando valor nutricional semelhantes. Os níveis de aflatoxina nas rações de peixe foram inferiores ao limite permitido pela legislação brasileira. A detecção de fumonisina (n.d. a $11,22 \mu \mathrm{g} / \mathrm{g}$ ) na maioria das rações, confirmou unanimidade do emprego de milho como ingrediente. Não houve diferença entre ração peletizada e extrusada quanto ao nível destas micotoxinas, porém a co-ocorrência aflatoxina e fumonisina em 3 marcas de rações (A, B e E), sugere que apesar de baixo nível, a ingestão simultânea poderia resultar no risco de sinergismo tóxico. A co-ocorrência indica a importância do monitoramento de micotoxinas no controle de qualidade da dieta de peixes, devido à escassez de informações sobre riscos de bioacumulação na cadeia alimentar.

\section{Agradecimentos}

Ao Conselho Nacional de Pesquisa e Desenvolvimento Científico e Tecnológico (CNPq) e Fundação Araucária-PR, pelo financiamento da pesquisa. Ao CNPq, também pela quota de Bolsas, referente a Produtividade, Apoio Técnico e Iniciação Científica a Elisa Y. Hirooka. À Coordenação de Aperfeiçoamento de Pessoal de Nível Superior (CAPES/MEC) pela concessão de bolsa de Mestrado à Elisabete H. Hashimoto. 


\section{Referências Bibliográficas}

ABOUZIED, M.M. \& PESTKA, J.J. Simultaneus screening of fumonisin B1, Aflatoxin B1, and Zearalenone by Line Immunoblot: A computer-assisted multianalyte assay system. Journal of the Association of Official Analitical Chemists, Washington,v.77, n.2, p.495-500, 1994.

ADCP-FAO ADCP/REP/87 - Aquaculture Developing and Control Programe 1987. Food and Agriculture of the United Nation. Geneva, 1987. Disponível em: <http:// www.fao.org/docrep>. Acesso em: jun.2002.

ARAGÃO, W. Piscicultura brasileira. Disponível em: <http://tudoparana.globo.com/gazetadopovo/arquivo>. Acesso em: jun. 2002.

BRASIL. Ministério da Agricultura, Pecuária e Abastecimento. Departamento de Pesca e Aqüicultura. Coordenação de Pesca e Aqüicultura - Relatórios. Disponível e, <http.www.agricultura.gov.br/dpa>. Acesso em jan.2001.

BUSSER, M.D. \& ABBAS, H.K. Effects of exrtusion temperature and dwell time on aflatoxin levels in cottonseed. Journal of Agricultural and Food Chemisry. Washington, v.50, n.9, p.25556-25569, 2002.

CAZZANIGA, D., BASILICO, J.C., GONZÁLEZ, R.J. TORES, R.L., GREEF, D.M. Mycotoxinns inactivation by extrusion cooking of corn flour. Letter in Applied Microbiology, Oxford, v.33, n.2, p.144-147, 2001.

CREPPY, E.E. Update of survey, regulation and toxic effects of mycotoxin in Europe. Toxicology Letters. Amsterdan, v.127, p-19-28, 2002.

EL-SAYED, A.F.M. Alternative dietary protein sources for farmed tilapia, Oreochromis spp. Aquaculture, Amsterdan, v.179, p.149-168, 1999.

EMATER - Empresa de Assistência Técnica e Extensão Rural. Piscicultura - Paraná. Disponível em <http:// www.emater.gov.pr.br>. Acesso em jun. 2002.

FAO. Joint FAO/WHO Expert Commitee on Food Additives. Summary and Conclusions. Food and Agriculture of the United Nation. Geneva. Disponível em <http://www.fao.org>. Acesso em: fev.2001.

FAO. 'Aquaculture Developing and Control Program'ADCP/FAO Food and Agriculture of the United Nation. 1987.Geneva. Disponível em <http://www.fao.org>. Acesso em out.2000.

FURUYA, W.M. Nutrição de peixes. In: MOREIRA, H.L.M. VARGAS, L.; RIBEIRO.R.P., ZIMMERMANN, S. Fundamentos da Moderna Aqüicultura. Canoas: Editora da Ulbra. 2001, p.59-68.
HARRISON, L.R., COLVIN, B.M., GRENNE, J.T., NEWMAN, L.E., COLE, J.R. Pulmonary edema and hydrotorax in suine produced by fumonisin B1, a toxic metabolite of Fusarium moniliforme. Journal of Veterinary Diagnostic Investigation, Columbia, v.2, p.217-221, 1990.

HIROOKA, E.Y., YAMAGUCHI, M.M., AOYAMA, SUGIURA, Y., UENO, Y. The natural occurrence of fumonisin in Brazilian corn kernels. Food Additives Contaminants, New York v.13, p.173-183, 1996.

HOLANDA, E.V J; RIBEIRO, L.P; ALI,V.B.R; HOLANDA, E.D., MIRANDA, M.O.T.; Análise de viabilidade financeira de projetos de piscicultura. Informe Agropecuário. Belo Horizonte. v.21, n.203, p.10-15, 2000.

IARC-International Agency for Research on Cancer. Working Group on the Evaluation of carcinogenic risks to man,. Lyons. Geneva: World Health Organization. 1993, v.56

INSTITUTO ADOLFO LUTZ. Normas analiticas do Instituto Adolfo Lutz. 3 ed. São Paulo: Instituto Adolfo Lutz, 1985, v.1.

JACKSON, L.S, HLYWKA, J.J., SENTHIL, K.R, BULLERMAN, L.B. Effect of thermal proccessing on the stability of fumonisins Advances in Experimental Medicine and Biology, New York, v. 392, p.345-353, 1996.

KUBITZA, F. \& CYRINO J.E.P. Effects of feed Quality and Feeding practices on the Quality of fish; A Brazilian Fish Culture Outlook. In CHANG Y.K. \& WANG, S.S. (ed.) Advances in Extrusion Technology. Lancaster: Technomic Publishing Company, 1999, p.53-69.

LALL, S.P., The minerals. In: HALVER, J.E. (Ed). Fish Nutrition. 2 ed. San Diego: Academic Press, 1988. P.220-252.

MARASAS, W.F.O. Fumonisins: Their implications for human and animal health. Natural Toxins, New York, v.3, p.193-198, 1995.

MEYER, S.P. Aquaculture: Current Status and Future Developments. In CHANG Y.K. \& WANG, S. S. (eds.) Advances in Extrusion Technology. Lancaster: Technomic Publishing Company, 1999, p.3-9.

MICOTOXINAS- Legislação sobre micotoxinas. Disponível em <http://www.micotoxinas.com.br/legisla $>$. Acesso em: fev.2001.

MUNKVOLD, G.P; DESJARDINS, A.E. Fumonisin in maize. Can we reduce their occurrence? Plant disease. Saint Paul, v81, n6., p556-565, 1997.

NORRED, W.P. \& VOSS, K.A. Toxicity and role of fumonisins in animal diseases and human esophageal cancer. J. Food protection, Des Mojes, v.57, p.522-527, 1994. 
ONO, E.Y.S. \& HIROOKA, E.Y. [2002] Hazards of Fusarium verticillioides, a mycotoxigenic fungus. In: Kushwaha, R.K.S. (ed.)Fungi in Human and Animal Health. Índia: Scientific Publishers. 2002, p.355-385.

ONO, E.Y.S.; SUGIURA, Y.; HOMECHIN, M.; KAMOGAE, M.; UENO Y.; HIROOKA, E.Y. Effect of climatic condition on natural mycoflora and fumonisin in freshly harvested corn of the State of Paraná, Brazil. Mycopathologia, Den Haag, v. 147, n3, p.139-148, 1999.

ONO, E.Y.S; SASAKI, E.Y.; HASHIMOTO, E.H; HARA, L.N; CORRÊA, B; ITANO, E.N; SUGIURA; UENO, Y; HIROOKA, E.Y. Post-harvest Storage of corn:effect of beginning moisture content on mycoflora and fumonisin contamination. Food Additives and Contaminants, London, v.19, n11, 1081-1090, 2002.

PLAKAS S.M., LOVELAND, P.M., BAILEY, G.S., WILSON, G.L. Tissue disposition and excretion of C14labelled aflatoxin B1 after oral administration in Chanel catfish. Food Chemistry. Toxicology, Kidlington, v.29, n.12, p.805-808, 1991.
SMITH J.E. \& MOSS, M.O. (Eds.). Mycotoxins; formation, Analysis and Significance. Chichester: John Wiley \& Sons Ltd. 148p. 1985.

SOARES, L.M.V. \& RODRIGUES-AMAYA, D.B., Métodos $\mathrm{p} /$ determinação simultânea de aflatoxina B e G, ocratoxina A, zearalenona e eterigmatocistina. Procedimento Operacional Padronizado. São Paulo, Instituto Adolfo Lutz. 1996.

UENO Y.; AOYAMA, S., SUGIURA, Y.; WANG,D-S.; HIROOKA, E.Y.; HARA, S. KARKI, T., CHEN, G., YU, SZ., A limited survey of fumonisin in corn-based products ins Asian countries. Mycotoxin Research, Alemanha, v.9, p.27-34, 1993.

WHO- World Health Organization. Toxicological and intake monograph on aflatoxins-- Food Additives Series, n40, 1998, Disponível em <http://www.who.int/fsf >. Acesso em jun. 2002. 